\title{
Diversity of Weed Flora in Citrus at ICAR-Regional Research Centre for Citrus, Biswanath Chariali, Assam, India
}

\author{
K. Kiran Kumar ${ }^{1,2 *}$ and P. Kiran Babu ${ }^{2}$ \\ ${ }^{1}$ Regional Research Centre for Citrus, ICAR-Central Citrus Research Institute, Biswanath \\ Chariali-784176, Assam, India \\ ${ }^{2}$ ICAR-Central Citrus Research Institute, Nagpur-440033, Maharashtra, India \\ ${ }^{3}$ ICAR-Indian Agricultural Research Institute, New Delhi-110012, India \\ *Corresponding author
}

\begin{abstract}
A B S T R A C T

\begin{tabular}{|l|}
\hline Key w or d s \\
$\begin{array}{l}\text { Diversity, Weed, } \\
\text { flora, Citrus, Assam }\end{array}$ \\
\hline Article Info \\
\hline $\begin{array}{l}\text { Accepted: } \\
\text { 15 March } 2019 \\
\text { Available Online: } \\
\text { 10 April } 2019\end{array}$ \\
\hline
\end{tabular}

Keywords

Diversity, Weed, , Assam

A systemic investigation was undertaken to assess the diversity of weed flora in citrus farm of Regional Research Centre for Citrus and inside the premises of Biswanath College of Agriculture, Biswanath Chariali, Assam during 2017-2018. A total of 134 weeds species belonging to 103 genera and 34 families were identified. Out of these, 104 species were dicotyldones and 30 species were monocotyldones. Among the 34 identified families, 9 families viz., poaceae, fabaceae, amaranthaceae, asteraceae, euphorbicaceae, cyperaceae, convolvulaceae, commilinaceae and solanaceae were represented by more than 5 weed species. Hibiscus moschetus was found with high Importance value index of 38.48 followed by Celosia argentea (33.18), Tribulus terrestris (32.57), Corchorus trilocularis (32.12), Mimosa pudica (31.25) and Euphorbia hirta (30.2). This is the first record of various weed species in citrus farm at RRCC.
\end{abstract}

\section{Introduction}

Weeds are one of the major deterrents in increasing the crop productivity as they compete with the crop for soil moisture and nutrients, which are the major limiting factors in horticultural farmyards (Anderson, 1983). The horticultural crops, especially young citrus plants are infested with a large number of weeds and increase the risk of insect and disease attack (Linares et al., 2008; Abbas and Fares, 2009; Onyegbule et al., 2014; Kaur and Rattanpal, 2017). Numerous exotic (non-

native) species were entered into India and invading the native flora. Few are introduced as ornamental or economical purposes and in this process seeds of many obnoxious weeds also got mixed up and firmly established and spread widely. Surprisingly the estimates put $18 \%$ of Indian flora as alien or non-native, of which about 55\% are American, $10 \%$ Asian, 20\% Asian and Malaysian and 15\% European and Central Asian species (Nayar, 1977). In case of citrus, weed flora causing 25-33\% yield loss to the farmers (Singh and Sharma, 2008). Therefore, efficient weed management 
becomes even more important in horticultural crops, especially in citrus. The losses due to weeds are more during rainy than the postrainy season. The nature and intensity of weed flora vary depending on agro-ecological conditions and management practices. To develop effective and economical weed management practices in citrus orchards, it is necessary to identify the weed flora, their nature, and intensity. Hence, the present investigation was undertaken to study the diversity of weeds in citrus at Regional Research Centre for Citrus (RRCC) under ICAR-Central Citrus Research Institute (ICAR-CCRI), Biswanath Chariali, Assam.

\section{Materials and Methods}

The study was carried out during 2017-2018 to identify major weed species in research farm of RRCC, ICAR-CCRI, and in the premises of Biswanath College of Agriculture (BNCA), Assam Agricultural University (AAU), Biswanath Chariali, Assam. The weed species encountered were carefully collected and identified using different morphological characters. Random quadrat method was adopted for studying phytosociological attributes of weeds. In each field site quadrat of $1 \mathrm{~m} \times 1 \mathrm{~m}$ was laid down in different locations of the campus and a sum of 20 quadrats for each location. These studies were carried in the rainy and post-rainy season.

Vegetation composition was evaluated by analyzing the frequency, density and Importance Value Index (IVI) according to Misra (1968) and Curtis and McIntosh (1950).

IVI (Importance Value Index) = Relative Density + Relative Frequency + Relative Dominance.

All the weeds from each quadrat were collected separately in polythene bags. Every specimen was carefully studied regarding vegetative and reproductive features. Provisional identification was made following 'Flora of British India' (Hooker 1872-1897) and other state, regional and local floras. All the plant families were arranged in sequence following Bentham and Hooker's classification (1862-83) with certain exceptions to accommodate recent modifications adopted after Cronquist (1968).

\section{Results and Discussion}

A total of 134 weeds species belonging to 103 genera and 34 families have been recorded from the study sites. Out of which 104 species belongs to dicotyledones and 30 species belongs to monocotyledons (Table 1). Among 34 families, nine families i.e. poaceae, fabaceae, amaranthaceae, asteraceae, euphorbicaceae, cyperaceae, convolvulaceae, commilinaceae and solanaceae were represented by more than 5 weed species (Fig. 1). A critical study on the flora of Assam and recent additions revealed the presence of 3895 species which are 2823 species of dicots under 190 families with 1006 genera + interspecific and 1072 species of monocots under 42 families with 332 genera + interspecific taxa. Out of which there are nearly $40 \%$ of the taxa considered as weeds in many crop fields (Kanjilal et al., 1931 and 1938).

Results depicted in Table 1 showed that Hibiscus moschatus (2.29 plants $/ \mathrm{m}^{2}$ ) followed by Corchorus trilocularis (2.23 plants $\left./ \mathrm{m}^{2}\right)$, Celosia argentia (2.11 plants $\left./ \mathrm{m}^{2}\right)$, Mimosa pudica (2.1 plants $\left./ \mathrm{m}^{2}\right)$, Euphorbia hirta (2.03 plants $\left./ \mathrm{m}^{2}\right)$ and Scoparia dulcis $\left(2\right.$ plants $\left./ \mathrm{m}^{2}\right)$ were dominant in the citrus farm. The Important Value Index of individual weed species reported that Hibiscus moschetus (38.48) is the most important species followed by Celosia argentea (33.18), Corchorus trilocularis (32.12), Tribulus terrestris 
(32.57), Mimosa pudica (31.25) and for Scoparia dulcis and Euphorbia hirta (30.2) in the citrus farm at RRCC, Biswanath Chariali. Deka and Baruah, 2015 reviewed the distribution of weeds in major agricultural, horticultural, forest and wetland ecosystems in North Eastern regions of India.

Table.1 Checklist of weed species with density (D) and Importance Value Index (IVI) found during 2017-2018 in citrus farm and surrounding premises at RRCC, Biswanath Chariali, Assam

\begin{tabular}{|c|l|l|c|c|}
\hline S.No & Scientific Name & Family & D & IVI \\
\hline $\mathbf{1}$ & Cleome aspera & Cleomaceae & 0.71 & 3 \\
\hline $\mathbf{2}$ & C. viscosa & Cleomaceae & 1.3 & 22.69 \\
\hline $\mathbf{3}$ & Hybanthus enneaspermus & Violaceae & 1.22 & 10.8 \\
\hline $\mathbf{4}$ & Polygala elongata & Polygalaceae & 1.08 & 6.45 \\
\hline $\mathbf{5}$ & Portulaca oleraceavar. Oleracea & Portulacaceae & 1.5 & 20.84 \\
\hline $\mathbf{6}$ & Abutilon indicum & & & \\
\hline $\mathbf{7}$ & Hibuscus moschatus & Malvaceae & 1.03 & 6.05 \\
\hline $\mathbf{8}$ & Sida acuta & Malvaceae & 2.29 & 38.48 \\
\hline $\mathbf{9}$ & S. cordata & Malvaceae & 1.15 & 16.93 \\
\hline $\mathbf{1 0}$ & Melochia corchorifolia & Malvaceae & 0.83 & 7 \\
\hline $\mathbf{1 1}$ & Waltheria indica & Sterculiaceae & 0.71 & 6.74 \\
\hline $\mathbf{1 2}$ & Corchorus aestuans & Sterculiaceae & 0.61 & 3.65 \\
\hline $\mathbf{1 3}$ & C. trilocularis & Tiliaceae & 0.65 & 7.34 \\
\hline $\mathbf{1 4}$ & Enicostema axillare & Tiliaceae & 2.23 & 33.12 \\
\hline $\mathbf{1 5}$ & Tribulus terrestris & Gentianaceae & 0.57 & 6.43 \\
\hline $\mathbf{1 6}$ & Alysicarpus bupleurifolius & Zygophyllaceae & 1.9 & 32.57 \\
\hline $\mathbf{1 7}$ & A. rugosus & Fabaceae & 1.1 & 5.3 \\
\hline $\mathbf{1 8}$ & Aeschynomene indica & Fabaceae & 0.82 & 9.65 \\
\hline $\mathbf{1 9}$ & Clitoria ternatea & Fabaceae & 1.2 & 10.6 \\
\hline $\mathbf{2 0}$ & Indigofera linifolia & Fabaceae & 0.28 & 5.61 \\
\hline $\mathbf{2 1}$ & I. linnaei & Fabaceae & 0.8 & 3.06 \\
\hline $\mathbf{2 2}$ & Macroptilium atropurpureum & Fabaceae & 1.3 & 5.17 \\
\hline $\mathbf{2 3}$ & Rhynchosia minima & Fabaceae & 0.34 & 0.96 \\
\hline $\mathbf{2 4}$ & Tephrosia pumila & Fabaceae & 0.72 & 3.01 \\
\hline $\mathbf{2 5}$ & T. purpurea & Fabaceae & 0.51 & 4.53 \\
\hline $\mathbf{2 6}$ & Vigna aconitifolia & Fabaceae & 0.59 & 10.12 \\
\hline $\mathbf{2 7}$ & Cassia pumila & Fabaceae & 0.56 & 2.73 \\
\hline $\mathbf{2 8}$ & Mimosa pudica & Caesalpiniaceae & 0.22 & 0.56 \\
\hline $\mathbf{2 9}$ & Ammania baccifera var. Baccifera & Mimosaceae & 2.1 & 31.06 \\
\hline $\mathbf{3 0}$ & Litrullus colycinthus & Cucurbitaceae & 0.7 & 2.23 \\
\hline & & & 10.49 \\
\hline
\end{tabular}




\begin{tabular}{|c|c|c|c|c|}
\hline 31 & Coccinia grandis & Cucurbitaceae & 0.56 & 4.27 \\
\hline 32 & Cucumis melo & Cucurbitaceae & 0.25 & 2.03 \\
\hline 33 & C. sativus & Cucurbitaceae & 0.42 & 3.9 \\
\hline 34 & Mollugo nudicaulis & Molluginaceae & 0.64 & 2.08 \\
\hline 35 & M. pentaphylla & Molluginaceae & 0.63 & 1.87 \\
\hline 36 & Centella asiatica & Apiaceae & 0.5 & 2.08 \\
\hline 37 & Borreria articularis & Rubiaceae & 0.58 & 5.03 \\
\hline 38 & B. pusilla & Rubiaceae & 1.1 & 14.21 \\
\hline 39 & Hedyotis corymbosa & Rubiaceae & 0.76 & 2.86 \\
\hline 40 & H. puberula & Rubiaceae & 0.82 & 3.21 \\
\hline 41 & Ageratum conyzoides & Asteraceae & 0.56 & 10.28 \\
\hline 42 & Bidens pilosa & Asteraceae & 0.92 & 10.4 \\
\hline 43 & Blumea mollis & Asteraceae & 0.6 & 1.84 \\
\hline 44 & Conyza ambigua & Asteraceae & 0.61 & 5.42 \\
\hline 45 & Echinops echinatus & Asteraceae & 0.55 & 4.71 \\
\hline 46 & Eclipta prostrata & Asteraceae & 0.65 & 5.52 \\
\hline 47 & Parthenium hysterophorus & Asteraceae & 1.41 & 15.16 \\
\hline 48 & Tridax procumbens & Asteraceae & 1.21 & 12.32 \\
\hline 49 & Vernonia cineria & Asteraceae & 1.09 & 12.08 \\
\hline 50 & Xanthium strumarium & Asteraceae & 0.19 & 0.93 \\
\hline 51 & Catheranthus pusillus & Apocyanaceae & 0.6 & 4.22 \\
\hline 52 & Calotropis gigantia & Asclepiadaceae & 0.25 & 2.02 \\
\hline 53 & C. procera & Asclepiadaceae & 0.26 & 1.89 \\
\hline 54 & Coldenia procumbens & Boraginaceae & 0.26 & 0.75 \\
\hline 55 & Heliotropium indicum & Boraginaceae & 0.71 & 2.08 \\
\hline 56 & H. ovalifolium & Boraginaceae & 0.66 & 3 \\
\hline 57 & Trichodesma indicum & Boraginaceae & 1.68 & 18.13 \\
\hline 58 & Convolvulus arvensis & Convolvulaceae & 0.59 & 6.37 \\
\hline 59 & Cuscuta reflexa & Convolvulaceae & 0.48 & 5.2 \\
\hline 60 & Evolvulus alsinoides & Convolvulaceae & 1.03 & 3.41 \\
\hline 61 & Ipomoea pes-tigridis & Convolvulaceae & 0.32 & 1.21 \\
\hline 62 & Merremia gangetica & Convolvulaceae & 1.03 & 4.47 \\
\hline 63 & M. tridentata & Convolvulaceae & 0.56 & 2.07 \\
\hline 64 & Datura metel & Solanaceae & 0.28 & 4.1 \\
\hline 65 & D. stramonium & Solanaceae & 0.21 & 0.95 \\
\hline 66 & Physalis minima & Solanaceae & 0.2 & 1.2 \\
\hline 67 & Solanum nigrum & Solanaceae & 0.23 & 2.02 \\
\hline 68 & S. surratens & Solanaceae & 1.02 & 10.28 \\
\hline
\end{tabular}




\begin{tabular}{|c|c|c|c|c|}
\hline 69 & Scoparia dulcis & Scrophulariaceae & 2 & 30.2 \\
\hline 70 & Striga asiatica & Scrophulariaceae & 0.56 & 2.85 \\
\hline 71 & S. gesneroides & Scrophulariaceae & 0.32 & 0.82 \\
\hline 72 & Verbascum chinense & Scrophulariaceae & 0.28 & 1.41 \\
\hline 73 & Sesamum alatum & Pedaliaceae & 0.22 & 0.91 \\
\hline 74 & Asystasia gangetica & Acanthaceae & 0.34 & 1.85 \\
\hline 75 & Indoneesiella echioides & Acanthaceae & 0.46 & 3.11 \\
\hline 76 & Lepidagathis cristata & Acanthaceae & 0.22 & 0.37 \\
\hline 77 & Rungia repens & Acanthaceae & 0.41 & 1.21 \\
\hline 78 & Hyptis suaveolens & Lamiaceae & 0.34 & 2.8 \\
\hline 79 & Leucas aspera & Lamiaceae & 0.67 & 3.08 \\
\hline 80 & L. cephalotes & Lamiaceae & 0.72 & 4.1 \\
\hline 81 & Ocimum americanum & Lamiaceae & 0.75 & 3.6 \\
\hline 82 & Boerhavia diffusa & Nyctaginaceae & 1 & 17.31 \\
\hline 83 & B. erecta & Nyctaginaceae & 0.82 & 3.21 \\
\hline 84 & Achyranthus aspera & Amaranthaceae & 0.3 & 3.87 \\
\hline 85 & Aerva javanica & Amaranthaceae & 0.15 & 0.41 \\
\hline 86 & A. lanata & Amaranthaceae & 0.37 & 1.93 \\
\hline 87 & Allmania nodiflora & Amaranthaceae & 0.55 & 4.12 \\
\hline 88 & Alternanthera pungens & Amaranthaceae & 0.92 & 2.1 \\
\hline 89 & A. sessilis & Amaranthaceae & 1.11 & 10.29 \\
\hline 90 & A. pungens & Amaranthaceae & 0.56 & 10.57 \\
\hline 91 & Amaranthus viridis & Amaranthaceae & 1.16 & 12.82 \\
\hline 92 & Celosia argentea & Amaranthaceae & 2.11 & 33.18 \\
\hline 93 & Digera muricata & Amaranthaceae & 1.02 & 28.77 \\
\hline 94 & Gomphrena serrata & Amaranthaceae & 0.49 & 2.8 \\
\hline 95 & Cardiospermum halicacabum & Sapindaceae & 0.49 & 5.2 \\
\hline 96 & Acalypha ciliata & Euphorbiaceae & 0.52 & 8.26 \\
\hline 97 & Acalypha indica & Euphorbiaceae & 0.3 & 2.17 \\
\hline 98 & Croton bonplandianum & Euphorbiaceae & 0.83 & 6.15 \\
\hline 99 & Chrozophora tinctoria & Euphorbiaceae & 0.92 & 16.58 \\
\hline 100 & Euphorbia geniculata & Euphorbiaceae & 1.3 & 22.56 \\
\hline 101 & Euphorbia hirta & Euphorbiaceae & 2.03 & 30.2 \\
\hline 102 & Phyllanthus amarus & Euphorbiaceae & 0.65 & 2.08 \\
\hline 103 & P. maderaspatensis & Euphorbiaceae & 0.34 & 0.8 \\
\hline 104 & Tragia involucrata & Euphorbiaceae & 0.55 & 2.21 \\
\hline 105 & Commelina benghalensis & Commelinaceae & 1.08 & 12.91 \\
\hline 106 & Cynotis axillaris & Commelinaceae & 0.42 & 6.12 \\
\hline
\end{tabular}




\begin{tabular}{|l|l|l|c|c|}
\hline $\mathbf{1 0 7}$ & Cynotis culculata & Commelinaceae & 0.59 & 7.28 \\
\hline $\mathbf{1 0 8}$ & Cyanotis fasciculata & Commelinaceae & 0.72 & 10.51 \\
\hline $\mathbf{1 0 9}$ & Tonningia axillaris & Commelinaceae & 0.82 & 7.02 \\
\hline $\mathbf{1 1 0}$ & $\begin{array}{l}\text { Cyperus compressusssp. } \\
\text { Compressus }\end{array}$ & Cyperaceae & 0.65 & 12.32 \\
\hline $\mathbf{1 1 1}$ & C. difformis & Cyperaceae & 0.55 & 2.9 \\
\hline $\mathbf{1 1 2}$ & C. rotundus & Cyperaceae & 0.58 & 4.8 \\
\hline $\mathbf{1 1 3}$ & C. iria & Cypraceae & 1.01 & 12.3 \\
\hline $\mathbf{1 1 4}$ & C. nutans & Cypraceae & 0.56 & 10.1 \\
\hline $\mathbf{1 1 5}$ & C. polystachyos & Cypraceae & 0.52 & 2.32 \\
\hline $\mathbf{1 1 6}$ & C. rotundus & Cypraceae & 0.75 & 3.56 \\
\hline $\mathbf{1 1 7}$ & C. tenuculmis & Cypraceae & 0.66 & 5.74 \\
\hline $\mathbf{1 1 8}$ & Apluda mutica & Poaceae & 1.08 & 7.16 \\
\hline $\mathbf{1 1 9}$ & Aristida hystrix & Poaceae & 0.72 & 2.84 \\
\hline $\mathbf{1 2 0}$ & Arundinella setosa & Poaceae & 0.81 & 4.25 \\
\hline $\mathbf{1 2 1}$ & Chloris inflata & Poaceae & 1.48 & 14.95 \\
\hline $\mathbf{1 2 2}$ & Chrysopogon fulvus & Poaceae & 0.75 & 3.19 \\
\hline $\mathbf{1 2 3}$ & Cynodon dactylon & Poaceae & 1.53 & 20.46 \\
\hline $\mathbf{1 2 4}$ & Heteropogon contortus & Poaceae & 0.84 & 3.42 \\
\hline $\mathbf{1 2 5}$ & Ischaemum indicum & Poaceae & 0.56 & 4.28 \\
\hline $\mathbf{1 2 6}$ & Iseilema laxum & Poaceae & 0.47 & 5.32 \\
\hline $\mathbf{1 2 7}$ & Panicum flavidum & Poaceae & 0.28 & 3.75 \\
\hline $\mathbf{1 2 8}$ & P. repens & Poaceae & 0.78 & 10.21 \\
\hline $\mathbf{1 2 9}$ & Paspalum disticum & Poaceae & 0.3 & 5.16 \\
\hline $\mathbf{1 3 0}$ & Perotis indica & Poaceae & 0.83 & 4.32 \\
\hline $\mathbf{1 3 1}$ & Phalaris minor & Poaceae & 0.62 & 4.08 \\
\hline $\mathbf{1 3 2}$ & Setaria verticillata & Poaceae & 0.67 & 3.08 \\
\hline $\mathbf{1 3 3}$ & Tragus roxburghii & Poaceae & 0.46 & 3.57 \\
\hline $\mathbf{1 3 4}$ & Urochloa panicoides & Poaceae & 0.26 & 0.82 \\
\hline & & & & \\
\hline
\end{tabular}


Fig.1 Proportion of weed species in each family presented as percentage to the total species collected from the citrus farm and other premises at RRCC, Biswanath Chariali, Assam

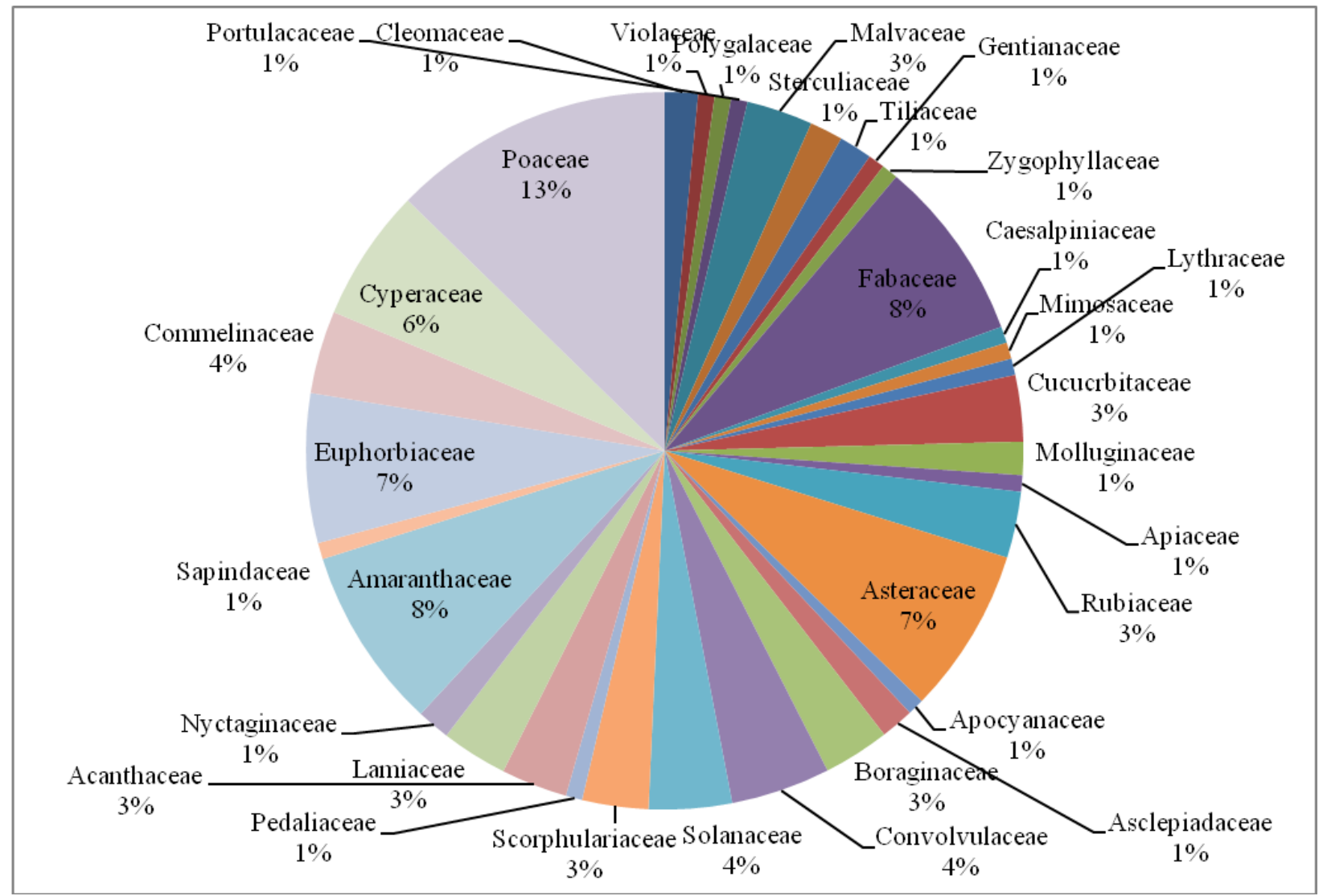

In conclusion, the present study aimed to investigate the diversity of weed flora in citrus farm of RRCC and in the premises of BNCA. The number of weed species reported in the study was relatively high. It is recommended that intensive ecological studies should be carried out to better understanding the drivers of the weeds species distribution and diversity. This necessary information will be useful for future effective management of these undesirable plants.

\section{References}

Abbas, F. and Fares, A. 2009. Best management practices in citrus production. Tree For. Sci. Biotech. 3(Special issue 1): 1-11.
Anderson, WP. 1983. Weed crop competition, in weed science, Principles. 2nd ed. pp. 15-33. West Publishing Company, St. Paill minn, USA.

Bentham, G. and Hooker, J.D. 1862-1883. Genera Plantarum. 3 vols. London.

Cronquist, A.J. 1968. The evolution and classification of flowering plants. London.

Deka, J. and Barua, I.C. 2015. Problem weeds and their management in the North-East Himalayas. Indian J. Weed Sci. 47(3): 296-305.

Hooker, J.D. 1872-1897. Flora of British India. Vol. I-VII, Reevi \& Co. Ltd., London.

Misra, R. 1968. Ecology Workbook. Oxford and IBH Publishing Company, New Delhi, India. 
Kaur, N. and Rattanpal, H.S. 2017. Weed management in citrus orchards. Rashtriya Krishi 12: 122.

Linares, J., Scholberg, J., Boote, K., Chase, C.A., Ferguson, J.J. and McSorley, R. 2008. Use of the cover crop weed index to evaluate weed suppression by cover crops in organic citrus orchards. Hort Sci. 43:27-34.

Misra, R. 1968. Ecology Work Book. Oxford \& IBH Publishing Co. New Delhi pp. 244.

Nayar, M.P. 1977. Changing patterns of Indian flora. Bulletin of Botanical Survey of India 19:145-154.

Onyegbule, U.N., Okpara, S.C., Nwanguma,
E.I., Ngbede, S.O., Ibekwe, H.N. and Uwalaka, A.O. 2014. The effect of different weed management methods on the growth performance of rough lemon citrus rootstock (Citrus jambhiri (L) Lush) in Okigwe, Southeast, Nigeria. Sci-Afric J. Sci. Issues. Res. Essays 2(2): 80-82.

Singh, M. and Sharma, S.D. 2008. Benefits of Triazine herbicides and other weed control technology in citrus management," In: Lebaron, H.M., McFarland, J.E. and Burnside, O.C. (Eds.), The Triazine Herbicides-50 Years Revolutionizing Agriculture, Elsevier, San Diego, pp. 199-209.

\section{How to cite this article:}

Kiran Kumar, K. and Kiran Babu, P. 2019. Diversity of Weed Flora in Citrus at ICARRegional Research Centre for Citrus, Biswanath Chariali, Assam, India. Int.J.Curr.Microbiol.App.Sci. 8(04): 2100-2107. doi: https://doi.org/10.20546/ijcmas.2019.804.247 